\title{
An Experimental Study of the Viscosity of Polymer Melts at High Shear Rate
}

\author{
Fumio KURIHARA and Shinichi KIMURA \\ Plastics Research \& Development Laboratory, Japan Synthetic Rubber Co., \\ 100 Kawajiri-cho, Yokkaichi, Mie 510, Japan
}

(Received October 15, 1984)

\begin{abstract}
Effects of shear heating on the measurement of polymer melt viscosity was studied for AS and ABS copolymers where A, S, and B stand for acrylonitrile, styrene, and butadiene, respectively. Melt viscosity under the high shear rate $\dot{\gamma}$ in a range from $10^{2}$ to $10^{5} \mathrm{~s}^{-1}$ was observed by two slit dies of different thicknesses and connected to the head of the injection molding machine. Agreement between the data for the dies became poor with increasing shear rate and the discrepancy could not be neglected at shear rates higher than $10^{3} \mathrm{~s}^{-1}$. The discrepancy was assumed to be caused by shear heating and an attempt was made to correct the viscosity curve by taking this effect into account. By introducing an Arrhenius type relation for the temperature dependence of viscosity, fairly good agreement was obtained for measurement of viscosity with different dies. A single curve of the shear stress $\tau_{\mathrm{w}}$ as a function of $\dot{\gamma}$ was obtained.
\end{abstract}

KEY WORDS Melt viscosity / High Shear Rate / Shear Heating / Shear Stress / Acrylonitrile-Butadiene-Styrene Copolymer / Acrylonitrile-Styrene Copolymer /

Injection molding is a very important industrial process for manufacturing plastic products. The properties of molded products obtained by injection molding depend on material properties and processing conditions. For injection molding, viscosity over a wide range of shear rates from $10^{2}$ to $10^{5} \mathrm{~s}^{-1}$ is quite useful, since a very high shear rate is generated both at the nozzle of the injection molding machine and gate of the mold, resulting in significant temperature rise. Temperature rise due to shear heating must be taken into account in measuring viscosity at high shear rates. Although there are many reports ${ }^{1}$ on viscosity measurement in a low shear rate range, little data are available for a high shear rate range above $10^{3} \mathrm{~s}^{-1}$, because of the difficulty induced by shear heating during viscosity measurement. During the past several years, a number of studies ${ }^{2,3}$ have been published on temperature rise behavior. For example, Vlachopoulos and Keung ${ }^{4}$ solved the energy equation numerically for a power law fluid in a fully developed flow and presented relation in terms of the Nusselt number and bulk temperature rise. Cox, Macosko, ${ }^{5}$ and Ybarra $^{6}$ have investigated experimentally the surface temperature rise of $\mathrm{ABS}$ and polyethylene extruding through slits, with an infrared pyrometer. They solved numerically the mass, momentum and energy equations.

In the present paper, a method for determining the viscosity of polymer melts in a shear rate range of $10^{2}$ to $10^{5} \mathrm{~s}^{-1}$ by correcting the effect of shear heating is proposed. As shown later, a great temperature rise of $90^{\circ} \mathrm{C}$ or more is usually observed at a shear rate of $10^{5} \mathrm{~s}^{-1}$ and the viscosity of most polymer melts decreases with temperature rise. Such a shear heating effect cannot be neglected in determining viscosity.

\section{EXPERIMENTAL}

\section{Materials}

All measurements were made with an 
acrylonitrile-butadiene-styrene copolymer (ABS) containing $20 \%$ rubber and an acrylonitrile-styrene copolymer (AS), both prepared by Japan Synthetic Rubber Co. They are denoted as ABS-1 and AS-1, respectively hereafter. The melt indices of the ABS-1 and the AS- 1 were $1.1 \mathrm{~g} / 10 \mathrm{~min}$, and $1.6 \mathrm{~g} / 10 \mathrm{~min}$ at $220^{\circ} \mathrm{C} / 5 \mathrm{~kg}$ (ASTM D1238), respectively.

\section{Equipment}

A schematic diagram of the die section is shown in Figure 1. The die section was attached directly to the head of the injection molding machine. Polymer melt flows through a reservoir part $(\mathrm{A}, \mathrm{B}, \mathrm{C})$ to the slit die part (D). Two types of slit dies were prepared. Each had three pressure tap holes and three thermocouple tap holes along the axis in a longitudinal direction. The dimensions of the slit dies and positions of the sensors are given in Table I. Three sets of pressure transducers and thermocouples were mounted adjacent to the wall surface of each slit die.

The temperature of the reservoir part (B) was monitored by another thermocouple (E) and that of the whole die section was controlled by a cartridge heater $(F)$. Local temperatures at the die wall surface were observed by the three thermocouples. The temperature of the injected material from the slit die, denoted as the melt bulk temperature, was

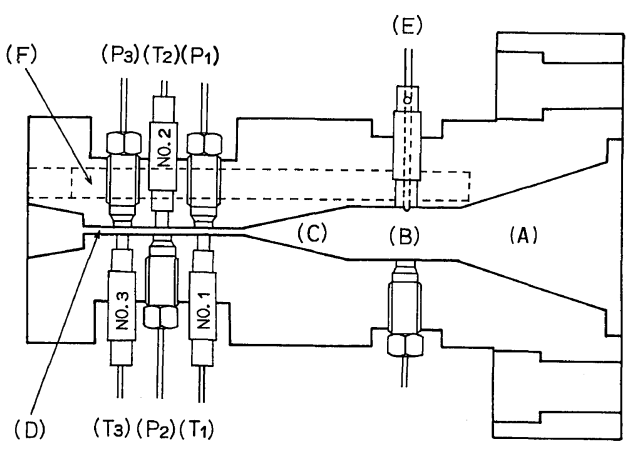

Figure 1. Sketch of the die section: (A), (B), and (C), reservoir; (D), slit die; $(\mathrm{E})$, thermocouple; $(\mathrm{F})$, cartridge heater; (T1), (T2), and (T3), thermocouples; (P1), (P2), and $(\mathrm{P} 3)$, pressure transducers. measured by another thermocouple.

The presssure transducers were calibrated against a dead load test and gave reproducible results within $\pm 0.5 \%$ of the full scale. They were of the strain-gage type and provided electrical outputs recorded on an oscillograph (SANEI-SOKKI Co.; visigraph 5L).

The injection molding machine used here was SYCAP (SUMITOMO-NESTAL Co.; N $515 / 150)$. The injection speed was well controlled by this injection machine within an error of $\pm 1 \%$. This accuracy was crucial to the results of the experiments.

\section{RESULTS AND DISCUSSION}

\section{Comparison of Slit Rheometer and Koka-Type Flow Tester Data}

In Comparing the results of slit dies with capillary rheometer data (used Koka-type Flow Tester), entrance correction ${ }^{7}$ was made, so as to confirm if the data of slit dies of two different aspect ratios agreed with capillary rheometer data. Apparent shear stress $\tau_{\mathrm{w}}$ and shear rate $\dot{\gamma}_{\mathrm{a}}$ at wall are defined as,

$$
\begin{array}{ll}
\tau_{\mathrm{w}}=\frac{R}{2}\left(\frac{\Delta P}{\Delta L}\right), & \dot{\gamma}_{\mathrm{a}}=\frac{4 Q}{\pi R^{3}} ; \text { capillary die } \\
\tau_{\mathrm{w}}=\frac{H}{2}\left(\frac{\Delta P}{\Delta L}\right), & \dot{\gamma}_{\mathrm{a}}=\frac{6 Q}{W H^{2}} ; \text { slit die }
\end{array}
$$

Table I. Slit die dimensions and pressure transducer and thermocouple locations

\begin{tabular}{lccc}
\hline & Unit & Slit die 1 & Slit die 2 \\
\hline Length of die (L) & $\mathrm{mm}$ & 45 & 45 \\
Slit thickness (H) & $\mathrm{mm}$ & 1 & 4 \\
Slit width (W) & $\mathrm{mm}$ & 10 & 10 \\
Aspect ratio (W/H) & & 10 & 2.5 \\
Location of pressure & & & \\
transducer and thermo- & & & \\
sensor from die inlet & & & \\
$\quad$ No. 1 & $\mathrm{mm}$ & 13 & 13 \\
No. 2 & $\mathrm{mm}$ & 26 & -26 \\
No. 3 & $\mathrm{mm}$ & 39 & 39 \\
\hline
\end{tabular}




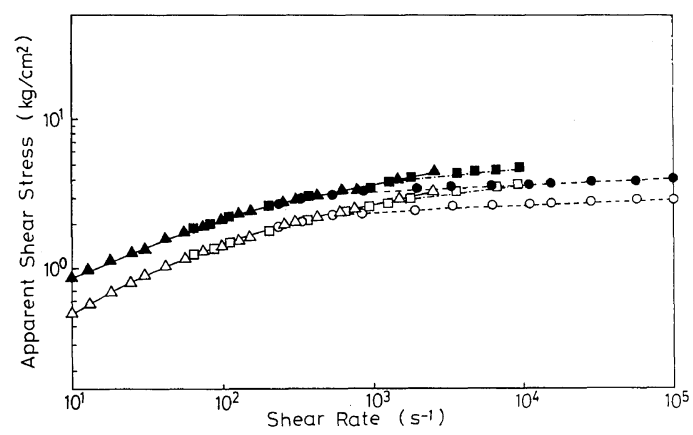

Figure 2. Apparent shear stress plotted against shear rate for AS-1 (unfilled mark) and ABS-1 (filled mark): Circles, squares and triangles represent results for slit die 1 , slit die 2 and the capillary rheometer, respectively.

where $(\Delta P / \Delta L)$ is the pressure gradient along the length of the die, $Q$ the volumetric flow rate, $R$ the radius of capillary die and $H$ and $W$ the depth and width of the slit die, respectively.

The results at $215^{\circ} \mathrm{C}$ are shown in Figure 2. Koka-type flow tester data $(\triangle)$ and two different slit rheometer data $(O, \square)$ are shown by unfilled and filled marks for AS-1 and ABS-1, respectively. The slit die with a large aspect ratio should be used to obtain reliable results. ${ }^{8}$ The aspect ratio of the slit die 2 was 2.5 and not large enough. In such a case, both the relation between $\tau_{\mathrm{w}}$ and $\Delta P$ and that between $\dot{\gamma}_{\mathrm{a}}$ and $Q$ must be modified, taking into account the flow pattern. Agreement between two slit die data and capillary die data, however, was fairly good at a shear rate lower than $10^{3} \mathrm{~s}^{-1}$. This agreement indicates that the slit dies in the present study may be considered equivalent to a capillary rheometer at a shear rate lower than $10^{3} \mathrm{~s}^{-1}$.

The extrudate was smooth at a low shear rate and became rough with increasing shear rate. Whether such surface roughness suggests a fracture in the flow through the die section remains to be clarified.

\section{Comparison of Results with Two Different Slit Dies}

The experimental data on the shear stress $\tau_{\mathrm{w}}$ at three different temperatures are shown in

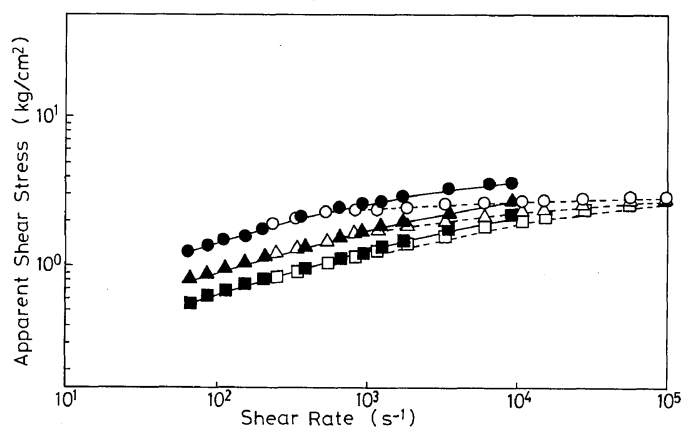

Figure 3. Apparent shear stress at various temperatures for AS-1: unfilled marks, slit die 1; filled marks, slit die 2; reservoir temperatures are 215,244 and $272^{\circ} \mathrm{C}$ from top to bottom.

Figure 3 for two slit dies. The unfilled and filled marks represent the results for thicknesses of $1 \mathrm{~mm}$ and $4 \mathrm{~mm}$, respectively. The agreement between the data of the dies with different thicknesses was good at a shear rate below $10^{3} \mathrm{~s}^{-1}$. It became poor with increasing shear rate, and the discrepancy could not be neglected. Differences in data will be discussed later on the basis of shear heating in relation to die thickness.

\section{Shear Heating in a Slit Die Flow}

The temperature rise in melt flow is generally considered to occur by two different mechanisms. One is change in the thermodynamic states of the polymer melt, so that temperature rise is calculated by the equation of state. However, the effect of pressure on temperature rise is assumed negligible in this study, since polymer melts may be treated as incompressible. The other is shear heating due to viscous dissipation.

The temperature rise in the flow was assumed due to shear heating. Figure 4 gives plots of bulk temperature rise as a function of shear rate for two slit dies, with the temperature rise defined by the difference between melt temperature measured with a CA type thermocouple at the die exit and that measured with thermocouple (E) at the reservoir in Figure 1. Data for a slit die 


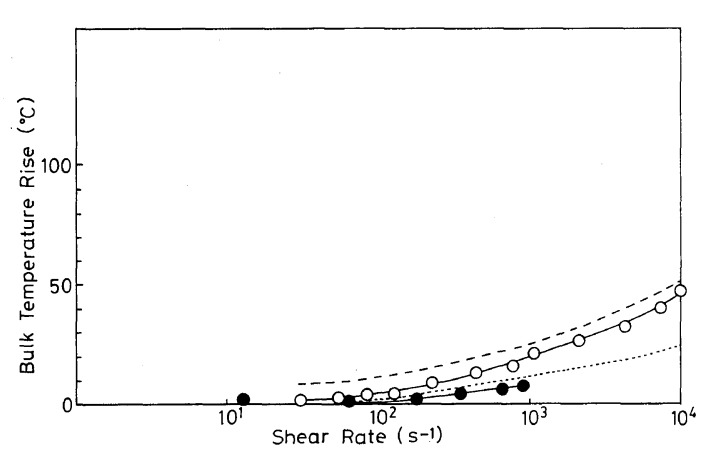

Figure 4. Bulk temperature rise plotted against shear rate for $\mathrm{AS}-1$ at $215^{\circ} \mathrm{C}$ of the reservoir: $\bigcirc$, slit die 1 ; slit die 2; broken and dotted lines represent calculated results with adiabatic and isothermal assumptions, respectively.

$1 \mathrm{~mm}$ in thickness $(\mathrm{O})$ and those $4 \mathrm{~mm}$ in thickness (O) were compared for sample AS-1.

The temperature rise in the former was much greater than that of the latter. The two lines in the figure are theoretical lines calculated according to Ybarra's presentation ${ }^{6}$ for slit die 1 . The broken line corresponds to the value determined on the basis of a adiabatic assumption, where heat flow through the die wall is negligible. The dotted line corresponds to the value made on an isothermal assumption, where the wall surface is infinitely conductive to heat flow and the wall temperature is equal to melt temperature at reservoir (B). The temperature rise measured on slit die 1 lies between the two calculated lines mentioned above. As expected, the experimental data are close to the line, according to the isothermal assumption at a low shear rate, and approach to the line of the adiabatic assumption with increasing shear rate.

The above discussion is concerned with bulk temperature rise measured at the exit of the die. The temperature rise, however, should be restricted to the wall surface of the die. Temperatures at the wall surface were measured by two thermocouples mounted adjacent to the walls as shown in Figure 1. The temperature rise at the wall, which is the difference between the measured temperature at the wall

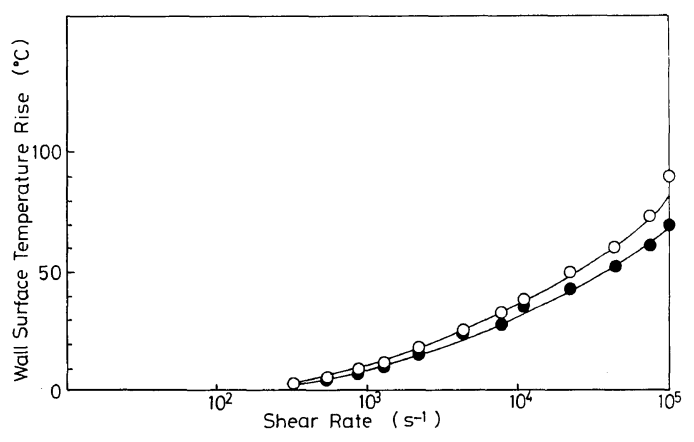

Figure 5. Wall surface temperature rise plotted against shear rate for AS-1 at a reservoir temperature of $215^{\circ} \mathrm{C}$; ○, thermocouple No. 1; O, thermocouple No. 2.

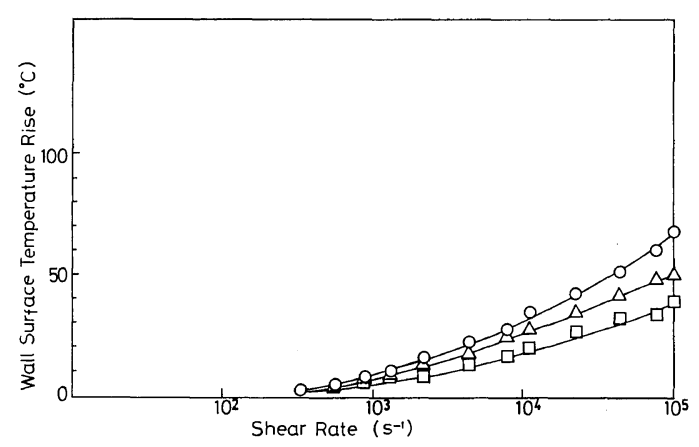

Figure 6. Wall surface temperature rise for $\mathrm{AS}-1$ at various reservoir temperatures: $\bigcirc, 215^{\circ} \mathrm{C} ; \triangle, 244^{\circ} \mathrm{C} ; \square$, $272^{\circ} \mathrm{C}$.

surface and that of the reservoir, is shown in Figure 5. The temperature rise at the center of the die (No. 2) is larger than that near the entrance (No. 1). As shown in Figures 4 and 5, the temperature rise due to shear heating must occur at a shear rate above $10^{3} \mathrm{~s}^{-1}$, and increased with shear rate. Figure 6 gives temperature rise at the wall surface (No. 2) plotted as a function of shear rate for AS-1 at three different temperatures of the reservoir.

The temperature rise at the wall surface increases with decreasing temperature. Consequently, the temperature rise is greater at the thinner slit (see Figure 4) at a lower temperature. Both can be explained as due to elevated stress level, as expected from Ybarra's work. The temperature rise at the wall surface is larger than the bulk temperature (compare 
Figure 4 with 6). This can be easily understood by considering the flow profile of the polymer melt through a die; that is, shear rate becomes maximum at the wall surface and is zero at the center of the die thickness.

\section{Viscosity Reduction Due to Shear Heating}

A considerable amount of viscosity reduction should be taken into account, since the temperature rises remarkably due to the shear heating effect near the die wall. Further, the shear rate at the wall surface $\dot{\gamma}$ may be altered, reflecting the rearrangement of the flow profile induced by temperature distribution in the die. In the present paper, rearrangement of flow profile was neglected and no correction made for the shear rate. A procedure for correcting viscosity change due to temperature rise is proposed.

The dependence of the viscosity $\eta$ on temperature is assumed independent of the shear rate and expressed as,

$$
\eta(T)=\eta\left(T_{\mathrm{r}}\right) \cdot a_{\mathrm{T}}(T)
$$

where $T_{\mathrm{r}}$ is a reference temperature, and $a_{\mathrm{T}}(T)$, the shift factor given by an Arrhenius type

$$
a_{\mathrm{T}}(T)=\exp \left[\Delta H / R \cdot\left(1 / T-1 / T_{\mathrm{r}}\right)\right]
$$

wehre $\Delta H$ is the flow activation energy, and $R$, the universal gas constant. Equation 4 is usually applicable at zero shear viscosity, but is assumed valid at any shear rate in this paper. The same relation as eq 3 can be applied to shear stress $\tau(\dot{\gamma}, T)$, since $\tau(\dot{\gamma}, T)$ is equal to $\eta(\dot{\gamma}, T) \cdot \dot{\gamma}$. From eq 3 and 4 , the temperature dependence of $\tau(\dot{\gamma}, T)$ can be expressed as,

$$
\ln \tau(\dot{\gamma}, T)=\ln \tau\left(\dot{\gamma}, T_{\mathrm{r}}\right)+\Delta H / R \cdot\left(1 / T-1 / T_{\mathrm{r}}\right)
$$

When $T_{\mathrm{r}}$ is the temperature at the die wall surface and $T$, that of the reservoir $T_{\mathrm{s}}$, the above equation gives a temperature dependence of $\tau_{\mathrm{w}}$ the shear stress corrected to temperature $T_{\mathrm{s}}$, as follows:

$$
\ln \tau_{\mathrm{w}}=\ln \tau_{\mathrm{wa}}+\Delta H / R \cdot\left(1 / T_{\mathrm{s}}-1 / T_{\mathrm{a}}\right)
$$

where $\tau_{\mathrm{wa}}$ is the shear stress observed at tem-

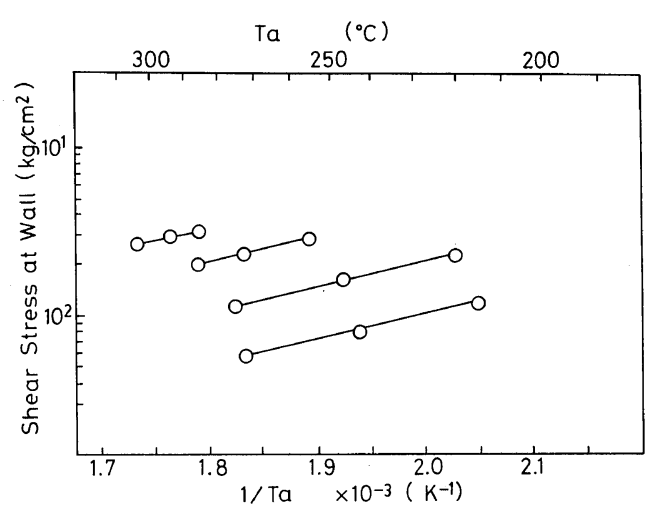

Figure 7. Dependence of apparent shear stress on temperature: shear rates are $1.1 \times 10^{5}, 1.1 \times 10^{4}, 8.5 \times 10^{2}$, and $6.8 \times 10^{1} \mathrm{~s}^{-1}$ from top to bottom.

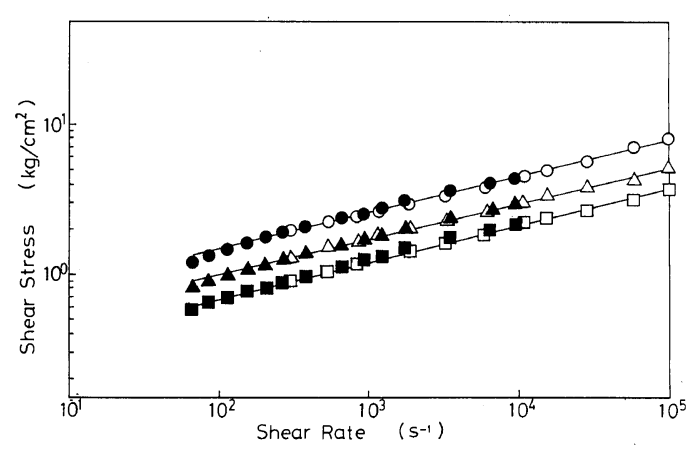

Figure 8. Shear stresses corrected by eq 6 plotted against shear rate for AS-1: filled and unfilled marks represent results for slit die 2 and slit die 1 , respectively; temperatures of the reservoir are 215, 244, and $272^{\circ} \mathrm{C}$ from top to bottom.

perature $T_{\mathrm{a}}$, the temperature at the die wall surface. Equation 4 is usually applied at zero shear viscosity in a homogeneous temperature field. In this case, the temperature profile is not homogeneous as a result of shear heating. The temperature at the die wall surface, however, was used as a trial.

Figure 7 gives $\ln \tau_{\text {wa }}$ plotted against $1 / T_{\mathrm{a}}$ at four different shear rates. It can be seen that the relation between the logarithmic shear stress $\tau_{\mathrm{wa}}$ and $1 / T_{\mathrm{a}}$ is linear and independent of the shear rate since the four lines corresponding to the respective shear rates are parallel. From the slopes, the activation energy $\Delta H$ was obtained. The value of the activation energy 
was $22 \mathrm{kcal} \mathrm{mol}^{-1}$, which agreed with the previous study. ${ }^{9}$ Putting this value into eq 6 , the decrease in shear stress due to temperature rise can be corrected. The plots in Figure 8 are values corrected by eq 6 for the data of Figure 3. In Figure 8 , the corrected shear stresses $\tau_{\mathrm{w}}$ for different slit dies with thicknesses of $1 \mathrm{~mm}$ and $4 \mathrm{~mm}$ are plotted on the same line for each temperature.

It may be reasonable to consider that the deviations of $\tau_{\text {wa }}$ in Figures 2 and 3 are responsible for temperature rise due to shear heating and that the relation between $\ln \tau_{w}$ and $\ln \gamma$ is approximately linear. That is, viscosity of the polymer melt obeys the power law in a shear rate range from $10^{2}$ to $10^{5} \mathrm{~s}^{-1}$. In the case of the other material ABS-1, where the activation energy $\Delta H$ was $20 \mathrm{kcal} \mathrm{mol}^{-1}$, good results were obtained by considering the temperature rise due to shear heating, as in the case of AS-1.

\section{CONCLUSION}

Melt viscosity in a high shear rate range from $10^{2}$ to $10^{5} \mathrm{~s}^{-1}$ was measured for an ABS and an AS resin by a slit die rheometer connected to the head of a injection molding machine. With increasing shear rate, a remarkable temperature rise was observed at the wall surface of the slit die. The shear stress $\tau_{\text {wa }}$ also behaved in a complicated manner, depending on the slit dimensions, set temperature, and shear rate. Reduction in shear stress at the die wall surface was assumed to be caused by temperature rise due to shear heating. An equation for correcting the shear heating effect was proposed by introducing an Arrhenius type relation for shear stress $\tau_{\mathrm{w}}$ :

$$
\ln \tau_{\mathrm{w}}=\ln \tau_{\mathrm{wa}}+\Delta H / R \cdot\left(1 / T_{\mathrm{s}}-1 / T_{\mathrm{a}}\right) .
$$

By this equation, correction of the data obtained with different slit demensions was made. A single curve of $\tau_{w}$ as a function of $\dot{\gamma}$ was obtained. The relation between shear stress $\tau_{w}$ and shear rate $\dot{\gamma}$ was found to approximate a power law in a high shear rate range from $10^{2}$ to $10^{5} \mathrm{~s}^{-1}$.

Acknowledgements. The authors express their sincere appreciation to Mr. Isamu Sakata for his technical assistance in carrying out the experiments. The authors also gratefully acknowledge the helpful comments of Drs. Mitsuo Abe, Yutaka Obata, and Kazuhiko Ninomiya.

\section{REFERENCES}

1. C. D. Han, J. Appl. Polym. Sci., 15, 2567 (1971).

2. H. T. Kim and E. A. Collins, Polym. Eng. Sci., 11, 83 (1971).

3. E. J. Novotny and R. E. Eckert, Trans. Soc. Rheol., 17, 222 (1973).

4. J. Vlachopoulos and C. K. J. Keung, AIChE J., 18, 1272 (1972).

5. H. W. Cox and C. W. Macosko, AIChE J., 20, 785 (1974).

6. R. M. Ybarra and R. E. Eckert, AIChE J., 26, 751 (1980).

7. E. B. Bagley, J. Appl. Phys., 28, 624 (1957).

8. C. D. Han, "Rheology in Polymer Processing," Academic Press, New York, N.Y., 1976.

9. H. Kubota, J. Appl. Polym. Sci., 19, 2299 (1975). 